\title{
AN INVARIANT OF CONFORMAL MAPPINGS
}

\author{
BANG-YEN CHEN $^{1}$
}

ABSTRACT. A result of W. Blaschke on conformal invariants of a surface is generalized.

1. Introduction. A conformal mapping on euclidean $m$-space $E^{m}$ can be decomposed into a product of similarity transformations and inversions $\left\{\pi_{j}\right\}$ (Haantjes [2]). Let $M$ be a surface in $E^{m}$. If the center of inversion $\pi_{i}$ of the conformal mapping does not lie on the surface $M$ for all $\pi_{i}$, then the conformal mapping is called a conformal mapping of $E^{m}$ with respect to $M$. A quantity on $M$ is called a conformal invariant if it is invariant under conformal mappings of $E^{m}$ with respect to $M$.

The main purpose of this note is to prove the following

THEOREM. Let $M$ be a surface in $E^{m}$ with Gauss curvature $K$, mean curvature $H$ and volume element $d V$. Then $\left(H^{2}-K\right) d V$ is a conformal invariant.

If the codimension is one, this theorem was given by Blaschke [1].

2. Proof of the Theorem. It is obvious that the quantity $\left(H^{2}-K\right) d V$ is invariant under similarity transformations (motions and homothetics on $\left.E^{m}\right)$. Hence, it suffices to prove the Theorem for inversions. Let $\pi$ be an inversion on $E^{m}$ such that the center of $\pi$ does not lie on the surface $M$. We choose the origin at the center of the inversion $\pi$. Let $x$ and $\bar{x}$ be the position vectors of the origin surface $M$ and the inverse surface $\bar{M}$ respectively, and let $c$ be the radius of inversion $\pi$. Then we have

$$
\overline{\boldsymbol{x}}=\left(c^{2} / r^{2}\right) \boldsymbol{x}, \quad r^{2}=\boldsymbol{x} \cdot \boldsymbol{x} .
$$

From this we find that

$$
\begin{aligned}
d \bar{x} & =\left(c^{2} / r^{2}\right) d x-\left(2 c^{2} / r^{3}\right)(d r) x, \\
d \bar{x} \cdot d \bar{x} & =\left(c^{4} / r^{4}\right) d x \cdot d x .
\end{aligned}
$$

Received by the editors January 16, 1973.

AMS (MOS) subject classifications (1970). Primary 53A05, 53B25, 53C40.

Key words and phrases. Conformal mappings, inversion, conformal invariant, mean curvature, Guass curvature.

${ }^{1}$ This work was supported in part by NSF under Grant GP-36684.

(c) American Mathematical Society 1973 
Hence the volume element $d \bar{V}$ of $\bar{M}$ is given by

$$
d \bar{V}=\left(c^{4} / r^{4}\right) d V .
$$

Let $e_{3}, \cdots, e_{m-2}$ be any $m-2$ mutually orthogonal unit normal local vector fields on $M$. Then

$$
\overline{\boldsymbol{e}}_{\alpha}=\left(2\left(x \cdot \boldsymbol{e}_{\alpha}\right) / r^{2}\right) x-e_{\alpha}, \quad \alpha=3, \cdots, m-2,
$$

are $m-2$ mutually orthogonal unit normal vector fields on $\bar{M}$. From (2) and (5), we obtain

$$
d \overline{\boldsymbol{x}} \cdot d \overline{\boldsymbol{e}}_{\alpha}=\left(2 c^{2}\left(\boldsymbol{x} \cdot \boldsymbol{e}_{\alpha}\right) / r^{4}\right) d \boldsymbol{x} \cdot d \boldsymbol{x}-\left(c^{2} / r^{2}\right) d \boldsymbol{x} \cdot d \boldsymbol{e}_{\alpha} .
$$

Combining (3) and (6), we find that, for any unit vector $e$ of $M$ in $E^{m}$, the principal curvatures $k_{i}(e), i=1,2$, of $M$ with respect to $e$ satisfy the following

$$
\bar{k}_{i}(\overline{\boldsymbol{e}})=-\left(r^{2} / c^{2}\right) k_{i}(\boldsymbol{e})-\left(2 r^{2} / c^{2}\right)(\boldsymbol{x} \cdot \boldsymbol{e}), \quad i=1,2,
$$

where $\bar{k}_{i}(\bar{e})$ are the corresponding principal curvatures on $\bar{M}$ and $\bar{e}=\left(2(x \cdot e) / r^{2}\right) x-e$. Hence we obtain

$$
\begin{aligned}
& \left(\bar{k}_{1}(\bar{e})+\bar{k}_{2}(e)\right)^{2}-4 \bar{k}_{1}(\bar{e}) k_{2}(\bar{e}) \\
& =\left(r^{4} / c^{4}\right)\left\{\left(k_{1}(e)+k_{2}(e)\right)^{2}-4 k_{1}(e) k_{2}(e)\right\} .
\end{aligned}
$$

By taking averages of both sides of (8) over the spheres of unit normal vectors of $\bar{M}$ and $M$ at the corresponding points, we obtain

$$
\bar{H}^{2}-R=\left(r^{4} / c^{4}\right)\left(H^{2}-K\right),
$$

where $\bar{H}$ and $R$ are the mean curvature and the Gauss curvature of $\bar{M}$. Hence, from (4) and (9), we obtain the Theorem.

RemarK 1 . If $M$ is an orientable closed surface in $E^{m}$, then, by combining the Theorem and the well-known Gauss-Bonnet formula, we see that the integral $\int_{M} H^{2} d V$ is a global conformal invariant. If the codimension is one, this invariant was observed by White [3].

\section{REFERENCES}

1. W. Blaschke, Vorlesungen über Differentialgeometrie. III, Springer, Berlin, 1929.

2. J. Haantjes, Conformal representations of an n-dimensional euclidean space with a non-definite fundamental form on itself, Proc. Kon. Ned. Akad. Amsterdam 40 (1937), 700-705.

3. J. H. White, A global invariant of conformal mappings in space, Proc. Amer. Math. Soc. 38 (1973), 162-164.

Department of Mathematics, Michigan State University, East lansing, MichigAN 48823 